Prepared in cooperation with West Virginia Department of Transportation, Division of Highways

\title{
Generalized Skew Coefficients of Annual Peak Flows for Rural, Unregulated Streams in West Virginia
}

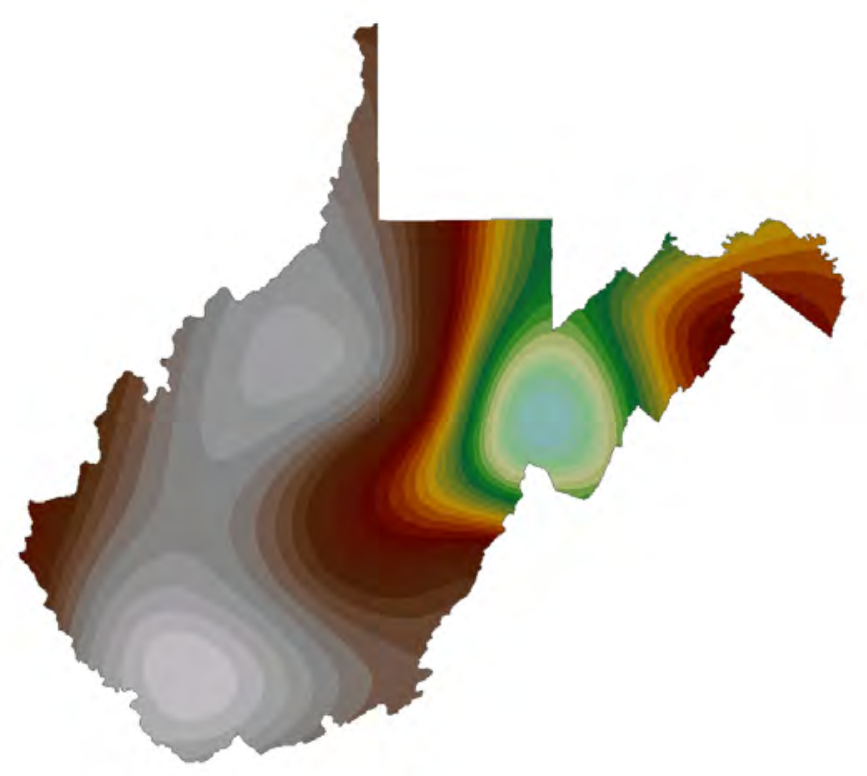

Open-File Report 2008-1304 
This page intentionally left blank. 


\section{Generalized Skew Coefficients of Annual Peak Flows for Rural, Unregulated Streams in West Virginia}

By John T. Atkins, Jr., Jeffrey B. Wiley, and Katherine S. Paybins

Prepared in cooperation with West Virginia Department of Transportation, Division of Highways

Open-File Report 2008-1304 


\section{U.S. Department of the Interior DIRK KEMPTHORNE, Secretary}

\section{U.S. Geological Survey \\ Mark D. Myers, Director}

\section{U.S. Geological Survey, Reston, Virginia: 2009}

For product and ordering information:

World Wide Web: http://www.usgs.gov/pubprod

Telephone: 1-888-ASK-USGS

For more information on the USGS--the Federal source for science about the Earth, its natural and living resources, natural hazards, and the environment:

World Wide Web: http://www.usgs.gov

Telephone: 1-888-ASK-USGS

Any use of trade, product, or firm names is for descriptive purposes only and does not imply endorsement by the U.S. Government.

Although this report is in the public domain, permission must be secured from the individual copyright owners to reproduce any copyrighted materials contained within this report.

Suggested citation:

Atkins, J.T., Jr., Wiley, J.B., and Paybins, K.S., 2009, Generalized skew coefficients of annual peak flows for rural, unregulated streams in West Virginia: U.S. Geological Survey Open-File Report 2008-1304, 13 p. 


\section{Contents}

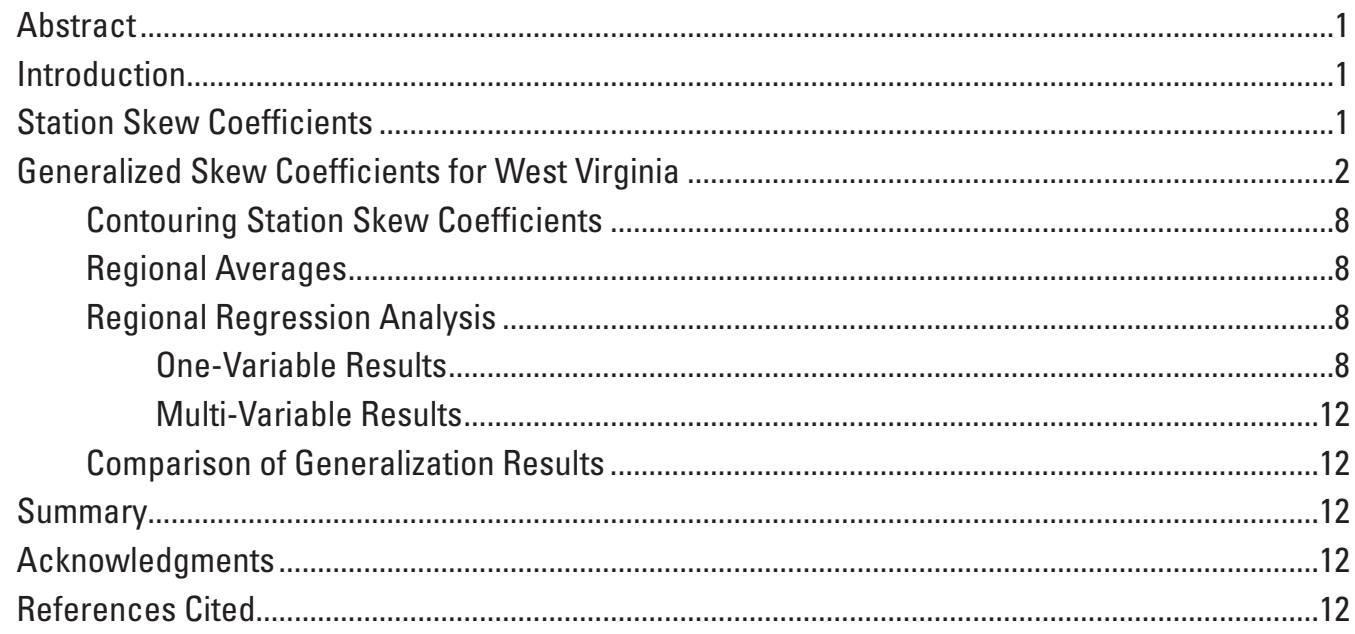

\section{Figures}

1. Map showing location of U.S. Geological Survey streamflow-gaging stations with 25 or more years of record in or near West Virginia ....................................................

2. Generalized skew map of West Virginia ...........................................................................

\section{Tables}

1. Station skews, differences between published and new (this study) flood-frequency computations of 100-year floods (reconciliation), and results of a Wilcoxon two-sample test of station skews for 147 streamflow-gaging stations in or near West Virginia with at least 25 years of record...............................................................................................

2. Average station skews and mean square errors for test regions using the 147 streamflow-gaging stations in or near West Virginia ..............................................................10

3. Results of screening relations of 38 basin variables to the generalized skew, determined from the West Virginia generalized skew map, for 147 streamflow-gaging stations using SAS procedure REG (with the "by $\mathrm{r}^{2 "}$ option). 


\section{Conversion Factors and Datums}

\begin{tabular}{lcl}
\hline \multicolumn{1}{c}{ Multiply } & \multicolumn{1}{c}{ By } & \multicolumn{1}{c}{ To obtain } \\
\hline \multicolumn{2}{c}{ Length } \\
\hline inch (in.) & 2.54 & centimeter $(\mathrm{cm})$ \\
inch (in.) & 25.4 & millimeter $(\mathrm{mm})$ \\
foot (ft) & 0.3048 & meter $(\mathrm{m})$ \\
mile (mi) & 1.609 & kilometer $(\mathrm{km})$ \\
\hline \multicolumn{3}{c}{ Area } \\
\hline square mile $\left(\mathrm{mi}^{2}\right)$ & 2.590 & square kilometer $\left(\mathrm{km}^{2}\right)$ \\
\hline \multicolumn{2}{c}{ Flow rate } & \\
\hline cubic foot per second $\left(\mathrm{ft}^{3} / \mathrm{s}\right)$ & 0.02832 & cubic meter per second $\left(\mathrm{m}^{3} / \mathrm{s}\right)$ \\
\hline
\end{tabular}

Temperature in degrees Fahrenheit $\left({ }^{\circ} \mathrm{F}\right)$ may be converted to degrees Celsius $\left({ }^{\circ} \mathrm{C}\right)$ as follows:

${ }^{\circ} \mathrm{C}=\left({ }^{\circ} \mathrm{F}-32\right) / 1.8$

Horizontal coordinate information is referenced to the North American Datum of 1983 (NAD 83). Intermediate results in the North American Datum of 1927 (NAD 27) were re-projected to NAD 83 using the NADCON option of ArcView 3.3. For assistance with conversions, the reader is directed to either the National Geodetic Survey website for NADCON at $h t t p: / / w w w . n g s . n o a a$. gov/TOOLS/Nadcon/Nadcon.html or the U.S. Army Corps of Engineers website for Corpscon at http://crunch.tec.army.mil/software/corpscon/corpscon.html.

Elevation, as used in this report, refers to distance above the vertical datum. 


\title{
Generalized Skew Coefficients of Annual Peak Flows for Rural, Unregulated Streams in West Virginia
}

\author{
By John T. Atkins, Jr., Jeffrey B. Wiley, and Katherine S. Paybins
}

\begin{abstract}
Generalized skew was determined from analysis of records from 147 streamflow-gaging stations in or near West Virginia. The analysis followed guidelines established by the Interagency Advisory Committee on Water Data described in Bulletin 17B, except that stations having 50 or more years of record were used instead of stations with the less restrictive recommendation of 25 or more years of record. The generalized-skew analysis included contouring, averaging, and regression of station skews. The best method was considered the one with the smallest mean square error (MSE). MSE is defined as the following quantity summed and divided by the number of peaks: the square of the difference of an individual logarithm (base 10) of peak flow less the mean of all individual logarithms of peak flow. Contouring of station skews was the best method for determining generalized skew for West Virginia, with a MSE of about 0.2174 . This MSE is an improvement over the MSE of about 0.3025 for the national map presented in Bulletin 17B.
\end{abstract}

\section{Introduction}

In the United States, the log-Pearson Type III distribution is widely used to calculate flood recurrences by fitting it to annual series of observed peak flows. This generally accepted method was published in Bulletin 17B by the Interagency Advisory Committee on Water Data (IACWD) (1982). Floodfrequency results are sensitive to the fit parameter, "skew," so Bulletin 17B includes guidelines for studies of skew (Interagency Advisory Committee on Water Data, 1982). Bulletin 17B contains a nationwide map of the generalized skew that has been used to determine skews in West Virginia. However, Bulletin 17B recommends that users develop generalized skew coefficients for their area of interest using the procedures outlined in the Bulletin. Generalized skews are to be developed using a minimum of 40 streamflow-gaging stations with 25 or more years of record. This is now possible for West Virginia because sufficient data have been collected in or near the State.
In an effort to provide updated information for planning and design activities related to flood-frequency calculations, the U.S. Geological Survey (USGS), in cooperation with the West Virginia Department of Transportation, Division of Highways, conducted an investigation to develop generalized skew coefficients for West Virginia. The results of this study supercede the longstanding method in West Virginia of using the nationwide generalized skew-coefficient map in Bulletin 17B. This report (1) describes the development of a statewide generalized skew-coefficient map, (2) presents the methods used, and (3) summarizes error analyses. The new generalized skew-coefficient map is specific to magnitude and frequency analysis of peak flows in West Virginia only.

\section{Station Skew Coefficients}

Skewness is a measure of the degree of asymmetry of a distribution. As used in this report, the "coefficient of skewness," "skew coefficient," or simply "skew" is a parameter used to match a series of logarithms of annual peak flows recorded at a streamflow-gaging station to the log-Pearson Type III statistical distribution. Statistically, skew is defined as (1) a numerical measure or an index of the lack of symmetry in a frequency distribution, (2) a function of the third moment of magnitudes about their mean, and (3) the adjusted third moment divided by the cube of the standard deviation. The skew coefficient for a single streamflow-gaging station is termed the "station skew."

The log-Pearson Type III distribution requires computation of the first moment (mean), the second moment (standard deviation), and the third moment (skew) of the base 10 logarithms of the station's annual peak flows. In this case, the first, second, and third moments for a sample are computed from the following:

$$
\begin{gathered}
\bar{X}=(\Sigma X) / N \quad \text { (first moment, mean, eq. } 2 \text { of Bulletin 17B), } \\
S=\left\{\left[\Sigma X^{2}-(\Sigma X)^{2} / N\right] /[N-1]\right\}^{1 / 2} \quad(\text { second moment, standard } \\
\text { deviation, eq. 3b of Bulletin 17B), }
\end{gathered}
$$


and

$$
\begin{gathered}
\mathrm{G}=\left[\mathrm{N}^{2}\left(\Sigma \mathrm{X}^{3}\right)-3 \mathrm{~N}(\Sigma \mathrm{X})\left(\Sigma \mathrm{X}^{2}\right)+2(\Sigma \mathrm{X})^{3}\right] /\left[\mathrm{N}(\mathrm{N}-1)(\mathrm{N}-2) \mathrm{S}^{3}\right] \\
\text { (third moment, skew, eq. } 4 \mathrm{~b} \text { of Bulletin 17B), }
\end{gathered}
$$

where

$\mathrm{X}$ is the logarithm of an annual peak flow, in $\mathrm{ft}^{3} / \mathrm{s}$, at a station for a given year;

$\Sigma \mathrm{X}, \Sigma \mathrm{X}^{2}, \Sigma \mathrm{X}^{3}$ is the sum of $\mathrm{X}, \mathrm{X}^{2}, \mathrm{X}^{3}$ for $\mathrm{N}$ years, respectively;

$\overline{\mathrm{X}} \quad$ is the mean of the logarithms of annual peak flows, in $\mathrm{ft}^{3} / \mathrm{s}$;

$\mathrm{N}$ is the number of peak flows;

$\mathrm{S} \quad$ is the standard deviation of the logarithms of annual peak flows, in $\mathrm{ft}^{3} / \mathrm{s}$; and

$\mathrm{G}$ is the skew coefficient (station skew, unitless).

\section{Generalized Skew Coefficients for West Virginia}

The 88 selected streamflow-gaging stations on rural, unregulated streams in West Virginia and 59 similar and nearby stations were combined to yield 147 stations for analysis (table 1, fig. 1). Records for a subset of 75 stations having at least 50 years of record also were analyzed (filled symbols in figure 1). To obtain a consistent set of skew values for all sites, new flood-frequency computations were performed using the PeakFQ computer program, version 4.1 (February 2002) as described by Flynn and others (2006). Annual peak flows from previous compilations for the 147 stations were updated from NWISWeb, the Web interface of the U.S. Geological Survey (USGS) National Water Information System (U.S. Geological Survey, 2008). Skew values were verified by recalculating them in PeakFQ and then reconciled to published values. Where there were no published skew values, the published 100-year flood flows were reconciled (see flood reconciliation, in percent, in table 1); nine stations are flagged as having 100 -year flood values with more than a 1-percent difference, and values at all but two stations are within 2.3 percent of the 100-year flood flow. In this study, skew values were computed and used for Prater Creek at Vasant, Va., and Potomac River at Point of Rocks, Md., that failed to reproduce the previously published values of 100 -year flood flows. This was done so that station skew values were in all cases calculable from the available peak-flow series. Overall, reconciliation problems were fewer than expected.

Confidence in longer streamflow records is usually well justified and has resulted in the development of methods that give greater weight to longer records, such as that described by Tasker and Stedinger (1986). However, if there is a general trend in skew as a function of record length (not chronological time), shorter records might reasonably be rejected, because more confidence can be placed in longer records. A simple

step test for trend (same as a two-sample test) can be obtained by using a non-parametric statistical test comparing the means of shorter and longer records.

The mean of the skews for 72 stations with less than 50 years of record (0.0615) and the mean for 75 stations with 50 or more years of record (0.2449) differ by 0.1834 (see table 1, footnote 1). This difference in the skew would make an important change in any flood-frequency calculation; therefore, its statistical significance needs verification. Can dividing the data set this way be ignored? That is, is the data set homogeneous with respect to record length?

A Wilcoxon-Mann-Whitney rank-sum test was used to answer this question. The Wilcoxon-Mann-Whitney rank-sum test statistic is the sum of the ranks for observations from the smaller of the two samples (in "Wilcoxon two-sample test" at the end of table 1). The null hypothesis is that the means are equal or that the two samples are drawn from the same population. The alternate hypothesis is that the samples have different "locations" (in the rank of values). The inputs to the test are the smallest sum of squares of the ranks of skews, its expected value, the standard deviation, and a small adjustment (table 1, footnote 2). The approximate form of the rank-sum test is considered good if the number of values is more than 20. The SAS procedure NPAR1WAY (SAS Institute Inc., 1990) was used to compute the approximate Wilcoxon twosample test, yielding a p-value of 0.0445 (details in "Wilcoxon two-sample test" at the end of table 1), which is significant at the alpha $=0.05$ level. The null hypothesis is therefore rejected. In other words, there is only about a 4.45 -percent chance of observing a difference in mean station skew as large as 0.1834 if the two samples were from the same population. For enhanced reliability, (1) station skews for the group of 75 stations with 50 or more years of record were used to contour the generalized skew map and for regression, but were not the only basis for computing regional averages (as explained farther on); and (2) values for the 147 stations were used for all comparisons between methods.

Bulletin 17B recommends comparison of mean square errors (MSE) between station skews and generalized skews to select the best generalization method. In this study, MSE developed by contouring, averages, and regression analysis for the 147 streamflow-gaging stations were compared to select the best generalized method. MSE is equal to the "standard error of estimate" squared, as follows:

$$
\mathrm{MSE}=\Sigma\left(\mathrm{SK}_{\mathrm{ST}}-\mathrm{SK}_{\mathrm{GEN}}\right)^{2} / \mathrm{N},
$$

where

$$
\begin{array}{cl}
\mathrm{N} & \text { is the number of stations (147), } \\
\mathrm{SK}_{\mathrm{ST}} & \text { is the station skew (unitless), and } \\
\mathrm{SK}_{\mathrm{GEN}} & \text { is the generalized skew (unitless). }
\end{array}
$$

The nationwide generalized skew map (Interagency Advisory Committee on Water Data, 1982), which can be viewed on the Humbolt State University Web site at URL http://www.humboldt.edu/ geology/for_download/hydrology/ 
Table 1. Station skews, differences between published and new (this study) flood-frequency computations of 100-year floods (reconciliation), and results of a Wilcoxon two-sample test of station skews for 147 streamflow-gaging stations in or near West Virginia with at least 25 years of record.

[A rank with a decimal place indicates a tie with a station with the same skew value. Shading indicates station with 50 or more years of record. ${ }^{1} \mathrm{MD}$, Maryland; WV, West Virginia; VA, Virginia]

\begin{tabular}{|c|c|c|c|c|c|c|c|}
\hline $\begin{array}{c}\text { Map } \\
\text { number } \\
\text { (fig. 1) }\end{array}$ & $\begin{array}{l}\text { Station } \\
\text { number }\end{array}$ & Station name & State & $\begin{array}{l}\text { Years of } \\
\text { systematic } \\
\text { record }\end{array}$ & $\begin{array}{c}\text { Flood } \\
\text { reconciliation, } \\
\text { in percent }^{2}\end{array}$ & $\begin{array}{l}\text { Station } \\
\text { skew, } \\
\text { unitless }\end{array}$ & $\begin{array}{c}\text { Rank of } \\
\text { station } \\
\text { skew }\end{array}$ \\
\hline 1 & 01595000 & North Branch Potomac River at Steyer & MD & 36 & 0 & 0.400 & 101.5 \\
\hline 2 & 01595300 & Abram Creek at Oakmont & WV & 27 & 0 & 0.355 & 96.5 \\
\hline 3 & 01595500 & North Br. Potomac River at Kitzmiller & MD & 41 & 0 & 1.633 & 147 \\
\hline 4 & 01596000 & North Br. Potomac River at Bloomington & $\mathrm{MD}$ & 25 & 0 & 0.378 & 99 \\
\hline 5 & 01596500 & Savage River near Barton & $\mathrm{MD}$ & 42 & 0 & 0.610 & 119 \\
\hline 6 & 01597000 & Crabtree Creek near Swanton & MD & 33 & 0 & 0.925 & 134 \\
\hline 7 & 01599000 & Georges Creek at Franklin & MD & 61 & 0 & 0.492 & 111 \\
\hline 8 & 01601500 & Wills Creek near Cumberland & MD & 61 & 0 & 0.983 & 137 \\
\hline 9 & 01603000 & N. Br. Potomac River near Cumberland & MD & 52 & 0 & 0.823 & 129 \\
\hline 10 & 01604500 & Patterson Creek near Headsville & WV & 59 & 0 & -0.264 & 33 \\
\hline 11 & 01605500 & South Branch Potomac River at Franklin & WV & 51 & 0 & 0.604 & 117 \\
\hline 12 & 01606000 & N. Fk. S. Br. Potomac River at Cabins & WV & 41 & 0 & 0.466 & 110 \\
\hline 13 & 01606500 & S. Br. Potomac River near Petersburg & WV & 70 & 0 & 0.961 & 136 \\
\hline 14 & 01607500 & S. Fk. S. Br. Potomac R. at Brandywine & WV & 54 & 0 & 0.777 & 127 \\
\hline 15 & 01608000 & S. Fk. S. Br. Potomac R. near Moorefield & WV & 68 & 0 & 0.898 & 132 \\
\hline 16 & 01608500 & S. Br. Potomac River near Springfield & WV & 74 & 0 & 0.667 & 122 \\
\hline 17 & 01609500 & Sawpit Run near Oldtown & MD & 25 & -0.5 & -0.760 & 5 \\
\hline 18 & 01610000 & Potomac River at Paw Paw & MD & 46 & 0.2 & -0.473 & 18 \\
\hline 19 & 01611500 & Cacapon River near Great Cacapon & WV & 74 & 0 & -0.074 & 54.5 \\
\hline 20 & 01613000 & Potomac River at Hancock & MD & 65 & 0 & 0.502 & 112 \\
\hline 21 & 01613900 & Hogue Creek near Hayfield & VA & 31 & -0.7 & -1.401 & 1 \\
\hline 22 & 01614000 & Back Creek near Jones Springs & WV & 46 & 0 & -0.531 & 14 \\
\hline 23 & 01615000 & Opequon Creek near Berryville & VA & 49 & 0.4 & 0.015 & 64 \\
\hline 24 & 01616500 & Opequon Creek near Martinsburg & WV & 50 & 0 & 0.176 & 78.5 \\
\hline 25 & 01617800 & Marsh Run at Grimes & MD & 27 & 0 & 0.584 & 116 \\
\hline 26 & 01618000 & Potomac River at Shepherdstown & MD & 64 & 1.4 & 0.288 & 86 \\
\hline 28 & 01620500 & North River near Stokesville & VA & 45 & 0 & 0.864 & 131 \\
\hline 29 & 01621000 & Dry River at Rawley Springs & VA & 31 & 0 & 1.365 & 145 \\
\hline 30 & 01621200 & War Branch near Hinton & VA & 28 & -0.4 & 0.392 & 100 \\
\hline 31 & 01622000 & North River near Burketown & VA & 63 & -0.8 & 0.945 & 135 \\
\hline 32 & 01632000 & N. Fk. Shenandoah R. at Cootes Store & VA & 69 & -0.9 & 0.087 & 68 \\
\hline 33 & 01632900 & Smith Creek near New Market & VA & 32 & -0.7 & -0.494 & 16 \\
\hline 34 & 01633000 & N. Fk. Shenandoah R. at Mount Jackson & VA & 48 & 0 & -0.108 & 50 \\
\hline 35 & 01633500 & Stony Creek at Columbia Furnace & VA & 32 & -2.1 & 0.073 & 67 \\
\hline 36 & 01634000 & N. Fk. Shenandoah River near Strasburg & VA & 65 & -0.2 & 0.461 & 108 \\
\hline 37 & 01634500 & Cedar Creek near Winchester & VA & 55 & -0.6 & -0.074 & 54.5 \\
\hline 38 & 01636500 & Shenandoah River at Millville & WV & 80 & 0 & 0.146 & 76 \\
\hline 39 & 01637000 & Little Catoctin Creek at Harmony & MD & 30 & 0 & 0.355 & 96.5 \\
\hline 40 & 01637500 & Catoctin Creek near Middletown & MD & 43 & 0.2 & 0.664 & 121 \\
\hline 41 & 01638500 & Potomac River at Point Of Rocks & MD & 96 & 9.8 & 0.187 & 80 \\
\hline
\end{tabular}


Table 1. Station skews, differences between published and new (this study) flood-frequency computations of 100-year floods (reconciliation), and results of a Wilcoxon two-sample test of station skews for 147 streamflow-gaging stations in or near West Virginia with at least 25 years of record.-Continued

[A rank with a decimal place indicates a tie with a station with the same skew value. Shading indicates station with 50 or more years of record. ${ }^{1}$ MD, Maryland; WV, West Virginia; VA, Virginia]

\begin{tabular}{|c|c|c|c|c|c|c|c|}
\hline $\begin{array}{c}\text { Map } \\
\text { number } \\
\text { (fig. 1) }\end{array}$ & $\begin{array}{l}\text { Station } \\
\text { number }\end{array}$ & Station name & State & $\begin{array}{c}\text { Years of } \\
\text { systematic } \\
\text { record }\end{array}$ & $\begin{array}{c}\text { Flood } \\
\text { reconciliation, } \\
\text { in percent }^{2}\end{array}$ & $\begin{array}{l}\text { Station } \\
\text { skew, } \\
\text { unitless }\end{array}$ & $\begin{array}{c}\text { Rank of } \\
\text { station } \\
\text { skew }\end{array}$ \\
\hline 42 & 01644000 & Goose Creek near Leesburg & VA & 65 & -1.2 & 0.523 & 114 \\
\hline 43 & 02011500 & Back Creek near Mountain Grove & VA & 34 & 0 & -0.445 & 20 \\
\hline 44 & 02012500 & Jackson River at Falling Spring & VA & 55 & 1.8 & -1.184 & 2 \\
\hline 45 & 02013000 & Dunlap Creek near Covington & VA & 58 & -0.7 & 0.369 & 98 \\
\hline 46 & 02014000 & Potts Creek near Covington & VA & 53 & -0.8 & -0.209 & 37 \\
\hline 47 & 03050000 & Tygart Valley River near Dailey & WV & 70 & 0 & 0.334 & 92 \\
\hline 48 & 03050500 & Tygart Valley River near Elkins & WV & 53 & 0 & 0.715 & 124 \\
\hline 49 & 03051000 & Tygart Valley River at Belington & WV & 90 & 0 & 0.289 & 87 \\
\hline 50 & 03051500 & Middle Fork River at Midvale & WV & 27 & 0 & 0.176 & 78.5 \\
\hline 51 & 03052000 & Middle Fork River at Audra & WV & 46 & 0 & 0.227 & 83.5 \\
\hline 52 & 03052500 & Sand Run near Buckhannon & WV & 51 & 0 & 0.402 & 103 \\
\hline 53 & 03053500 & Buckhannon River near Hall & WV & 83 & 0 & -0.349 & 27 \\
\hline 54 & 03054500 & Tygart Valley River at Phillipi & WV & 57 & 0 & 0.352 & 95 \\
\hline 55 & 03056500 & Tygart Valley River at Fetterman & WV & 32 & 0 & 0.251 & 85 \\
\hline 56 & 03057500 & Skin Creek near Brownsville & WV & 40 & 0 & -0.295 & 30 \\
\hline 57 & 03058000 & West Fork River at Brownsville & WV & 45 & 0 & 0.337 & 93 \\
\hline 58 & 03058500 & West Fork River at Butcherville & WV & 82 & 0 & -0.085 & 52 \\
\hline 59 & 03059000 & West Fork River at Clarksburg & WV & 60 & 0 & -0.488 & 17 \\
\hline 60 & 03059500 & Elk Creek at Quiet Dell & WV & 27 & 0 & -0.062 & 56 \\
\hline 61 & 03061000 & West Fork River at Enterprise & WV & 65 & 0 & -0.183 & 42 \\
\hline 62 & 03061500 & Buffalo Creek at Barrackville & WV & 73 & 0 & -0.178 & 43 \\
\hline 63 & 03062400 & Cobun Creek at Morgantown & WV & 32 & 0 & 0.854 & 130 \\
\hline 64 & 03062500 & Deckers Creek at Morgantown & WV & 30 & 0 & 0.324 & 91 \\
\hline 65 & 03065000 & Dry Fork at Hendricks & WV & 57 & 0 & 1.310 & 144 \\
\hline 66 & 03066000 & Blackwater River at Davis & WV & 76 & 0 & 0.746 & 125 \\
\hline 67 & 03069000 & Shavers Fork at Parsons & WV & 73 & 0 & 0.992 & 138 \\
\hline 68 & 03069500 & Cheat River near Parsons & WV & 84 & 0 & 1.144 & 141 \\
\hline 69 & 03070000 & Cheat River at Rowlesburg & WV & 74 & 0 & 0.796 & 128 \\
\hline 70 & 03070500 & Big Sandy Creek near Rockville & WV & 83 & 0 & 0.692 & 123 \\
\hline 71 & 03071000 & Cheat River near Pisgah & WV & 47 & 0 & 0.130 & 74 \\
\hline 72 & 03072000 & Dunkard Creek at Shannopin & PA & 35 & -0.8 & -0.277 & 31 \\
\hline 73 & 03075500 & Youghiogheny River near Oakland & MD & 49 & 0 & 0.095 & 69 \\
\hline 74 & 03076600 & Bear Creek at Friendsville & MD & 26 & 0 & 0.214 & 81.5 \\
\hline 75 & 03108000 & Raccoon Creek at Moffatts Mill & $\mathrm{PA}$ & 51 & 0 & -0.216 & 36 \\
\hline 76 & 03109000 & Lisbon Creek at Lisbon & $\mathrm{OH}$ & 35 & 0 & 0.441 & 106 \\
\hline 77 & 03109500 & Little Beaver Creek near East Liverpool & $\mathrm{OH}$ & 72 & 0 & 0.214 & 81.5 \\
\hline 78 & 03110000 & Yellow Creek near Hammondsville & $\mathrm{OH}$ & 47 & 0 & 0.296 & 88 \\
\hline 79 & 03111500 & Short Creek near Dillonvale & $\mathrm{OH}$ & 46 & -0.1 & -0.735 & 7 \\
\hline 80 & 03112000 & Wheeling Creek at Elm Grove & WV & 46 & 0 & -0.031 & 59 \\
\hline 81 & 03114000 & Captina Creek at Armstrongs Mills & $\mathrm{OH}$ & 38 & 0 & 0.061 & 66 \\
\hline
\end{tabular}


Table 1. Station skews, differences between published and new (this study) flood-frequency computations of 100-year floods (reconciliation), and results of a Wilcoxon two-sample test of station skews for 147 streamflow-gaging stations in or near West Virginia with at least 25 years of record.-Continued

[A rank with a decimal place indicates a tie with a station with the same skew value. Shading indicates station with 50 or more years of record. ${ }^{1} \mathrm{MD}$, Maryland; WV, West Virginia; VA, Virginia]

\begin{tabular}{|c|c|c|c|c|c|c|c|}
\hline $\begin{array}{c}\text { Map } \\
\text { number } \\
\text { (fig. 1) }\end{array}$ & $\begin{array}{l}\text { Station } \\
\text { number }\end{array}$ & Station name & State & $\begin{array}{l}\text { Years of } \\
\text { systematic } \\
\text { record }\end{array}$ & $\begin{array}{c}\text { Flood } \\
\text { reconciliation, } \\
\text { in percent }^{2}\end{array}$ & $\begin{array}{l}\text { Station } \\
\text { skew, } \\
\text { unitless }\end{array}$ & $\begin{array}{c}\text { Rank of } \\
\text { station } \\
\text { skew }\end{array}$ \\
\hline 82 & 03114500 & Middle Island Creek at Little & WV & 78 & 0 & 0.157 & 77 \\
\hline 83 & 03115600 & Barns Run near Summerfield & $\mathrm{OH}$ & 33 & 0 & 0.121 & 71 \\
\hline 84 & 03151500 & Little Kanawha River near Burnsville & WV & 35 & 0 & -0.427 & 22 \\
\hline 85 & 03152000 & Little Kanawha River at Glenville & WV & 69 & 0 & -0.008 & 62 \\
\hline 86 & 03153000 & Steer Creek near Grantsville & WV & 39 & 0 & -0.878 & 4 \\
\hline 87 & 03153500 & Little Kanawha River at Grantsville & WV & 69 & 0 & -0.354 & 25 \\
\hline 88 & 03154000 & W. Fk. Little Kanawha River at Rocksdale & WV & 63 & 0 & -0.200 & 38 \\
\hline 89 & 03154500 & Reedy Creek near Reedy & WV & 27 & 0 & -0.596 & 12 \\
\hline 90 & 03155000 & Little Kanawha River at Palestine & WV & 40 & 0 & -0.738 & 6 \\
\hline 91 & 03155500 & Hughes River at Cisco & WV & 59 & 0 & -0.092 & 51 \\
\hline 92 & 03171500 & New River at Eggleston & VA & 25 & 0 & 1.225 & 143 \\
\hline 93 & 03173000 & Walker Creek at Bane & VA & 54 & 0 & -0.302 & 29 \\
\hline 94 & 03175500 & Wolf Creek near Narrows & VA & 62 & 2.3 & -0.185 & 40.5 \\
\hline 95 & 03176500 & New River at Glen Lyn & VA & 82 & 0 & 1.006 & 139 \\
\hline 96 & 03178500 & Camp Creek near Camp Creek & WV & 29 & 0 & -0.122 & 49 \\
\hline 97 & 03179000 & Bluestone River near Pipestem & WV & 47 & 0 & -1.000 & 3 \\
\hline 98 & 03179500 & Bluestone River at Lilly & WV & 27 & 0 & -0.191 & 39 \\
\hline 99 & 03180000 & New River at Bluestone Dam & WV & 25 & 0 & 1.218 & 142 \\
\hline 100 & 03180500 & Greenbrier River at Durbin & WV & 54 & 0 & 1.624 & 146 \\
\hline 101 & 03182500 & Greenbrier River at Buckeye & WV & 68 & 0 & 0.609 & 118 \\
\hline 102 & 03183000 & Second Creek near Second Creek & WV & 29 & 0 & 0.107 & 70 \\
\hline 103 & 03183500 & Greenbrier River at Alderson & WV & 102 & 0 & -0.223 & 34.5 \\
\hline 104 & 03184000 & Greenbrier River at Hilldale & WV & 62 & 0 & 0.139 & 75 \\
\hline 105 & 03185000 & Piney Creek at Raleigh & WV & 31 & 0 & 0.340 & 94 \\
\hline 106 & 03186000 & New River at Fayette & WV & 35 & 0 & -0.434 & 21 \\
\hline 107 & 03186500 & Williams River at Dyer & WV & 68 & 0 & 0.522 & 113 \\
\hline 108 & 03187000 & Gauley River at Camden on Gauley & WV & 66 & 0 & 0.227 & 83.5 \\
\hline 109 & 03187500 & Cranberry River near Richwood & WV & 39 & 0 & 0.436 & 105 \\
\hline 110 & 03189000 & Cherry River at Fenwick & WV & 43 & 0 & 1.077 & 140 \\
\hline 111 & 03189100 & Gauley River near Craigsville & WV & 31 & 0 & -0.035 & 58 \\
\hline 112 & 03189500 & Gauley River near Summersville & WV & 37 & 0 & 0.912 & 133 \\
\hline 113 & 03190000 & Meadow River at Nallen & WV & 51 & 0 & 0.029 & 65 \\
\hline 114 & 03190400 & Meadow River near Mount Lookout & WV & 29 & 0 & -0.028 & 60.5 \\
\hline 115 & 03191500 & Peters Creek near Lockwood & WV & 31 & 0 & 0.400 & 101.5 \\
\hline 116 & 03192000 & Gauley River above Belva & WV & 69 & 0 & 0.444 & 107 \\
\hline 117 & 03193000 & Kanawha River at Kanawha Falls & WV & 120 & 0 & -0.075 & 53 \\
\hline 118 & 03194700 & Elk River below Webster Springs & WV & 68 & 0 & 0.769 & 126 \\
\hline 119 & 03195000 & Elk River at Centralia & WV & 29 & 0 & -0.043 & 57 \\
\hline 120 & 03197000 & Elk River at Queen Shoals & WV & 70 & 0 & 0.654 & 120 \\
\hline 121 & 03198500 & Big Coal River at Ashford & WV & 75 & 0 & 0.124 & 73 \\
\hline
\end{tabular}


Table 1. Station skews, differences between published and new (this study) flood-frequency computations of 100-year floods (reconciliation), and results of a Wilcoxon two-sample test of station skews for 147 streamflow-gaging stations in or near West Virginia with at least 25 years of record.-Continued

[A rank with a decimal place indicates a tie with a station with the same skew value. Shading indicates station with 50 or more years of record. ${ }^{1} \mathrm{MD}$, Maryland; WV, West Virginia; VA, Virginia]

\begin{tabular}{|c|c|c|c|c|c|c|c|}
\hline $\begin{array}{c}\text { Map } \\
\text { number } \\
\text { (fig. 1) }\end{array}$ & $\begin{array}{l}\text { Station } \\
\text { number }\end{array}$ & Station name & State & $\begin{array}{l}\text { Years of } \\
\text { systematic } \\
\text { record }\end{array}$ & $\begin{array}{c}\text { Flood } \\
\text { reconciliation, } \\
\text { in percent }^{2}\end{array}$ & $\begin{array}{c}\text { Station } \\
\text { skew, } \\
\text { unitless }\end{array}$ & $\begin{array}{c}\text { Rank of } \\
\text { station } \\
\text { skew }\end{array}$ \\
\hline 122 & 03199000 & Little Coal River at Danville & WV & 54 & 0 & -0.028 & 60.5 \\
\hline 123 & 03200500 & Coal River at Tornado & WV & 42 & 0 & -0.471 & 19 \\
\hline 124 & 03201000 & Pocatalico River at Sissonville & WV & 52 & 0 & -0.223 & 34.5 \\
\hline 125 & 03202000 & Raccoon Creek at Adamsville & $\mathrm{OH}$ & 68 & 0 & 0.314 & 89 \\
\hline 127 & 03203000 & Guyandotte River at Man & WV & 69 & 0 & -0.413 & 23 \\
\hline 128 & 03204000 & Guyandotte River at Branchland & WV & 76 & 0 & -0.637 & 10 \\
\hline 129 & 03204500 & Mud River near Milton & WV & 43 & 0 & 0.462 & 109 \\
\hline 130 & 03206600 & East Fork Twelvepole Creek near Dunlow & WV & 33 & 0 & -0.156 & 44.5 \\
\hline 131 & 03207000 & Twelvepole Creek at Wayne & WV & 31 & 0 & -0.138 & 47 \\
\hline 135 & 03208000 & Levisa Fork below Fishtrap Dam & KY & 55 & 0 & -0.134 & 48 \\
\hline 136 & 03208500 & Russel Fork at Haysi & VA & 65 & 2.2 & -0.375 & 24 \\
\hline 137 & 03208950 & Cranes Nest River near Clintwood & VA & 28 & -0.5 & 0.429 & 104 \\
\hline 138 & 03209000 & Pond R. blw. Flannagan Dam near Haysi & VA & 80 & 1.1 & 0.122 & 72 \\
\hline 139 & 03210000 & Johns Crek near Meta & KY & 46 & 0 & -0.541 & 13 \\
\hline 140 & 03212000 & Paint Creek at Staffordsville & KY & 32 & 0 & -0.615 & 11 \\
\hline 141 & 03213000 & Tug Fork at Litwar & WV & 56 & 0 & -0.276 & 32 \\
\hline 142 & 03213500 & Panther Creek near Panther & WV & 39 & 0 & -0.353 & 26 \\
\hline
\end{tabular}

Wilcoxon two-sample test

\begin{tabular}{|c|c|c|c|c|c|c|c|c|c|}
\hline \multicolumn{2}{|c|}{ Number of stations } & \multicolumn{2}{|c|}{ Sum of ranks } & \multicolumn{5}{|c|}{ Expected statistics under the null hypothesis } & \multirow[b]{2}{*}{ Conclusion } \\
\hline $\begin{array}{l}\text { With } 50 \\
\text { or more } \\
\text { years of } \\
\text { record }\end{array}$ & $\begin{array}{l}\text { With less } \\
\text { than } 50 \\
\text { years of } \\
\text { record }\end{array}$ & $\begin{array}{c}\text { With } 50 \\
\text { or more } \\
\text { years of } \\
\text { record }\end{array}$ & $\begin{array}{l}\text { With less } \\
\text { than } 50 \\
\text { years of } \\
\text { record }\end{array}$ & $\begin{array}{l}\text { Standard } \\
\text { deviation }\end{array}$ & $\begin{array}{c}\text { With } 50 \\
\text { or more } \\
\text { years of } \\
\text { record }\end{array}$ & $\begin{array}{l}\text { With less } \\
\text { than } 50 \\
\text { years of } \\
\text { record }\end{array}$ & $\begin{array}{c}\text { Normal } \\
\text { approximation } \\
\text { of Z-value }{ }^{3}\end{array}$ & $\begin{array}{c}\text { Probability } \\
\text { greater than } \\
\text { absolute } \\
\text { value of Z }\end{array}$ & \\
\hline
\end{tabular}

${ }^{1}$ Mean of station skews for shaded rows is 0.2449 and for unshaded rows is 0.0615 , for a difference of 0.1834 .

${ }^{2}$ Flood reconciliation, in percent $=100 *$ (published 100-year flood - recomputed 100-year flood $) /$ published 100-year flood.

${ }^{3} \mathrm{Z}=($ test statistic - expected + continuity correction $) /$ expected standard deviation $=(4,809-5,328+0.05) / 258.067=-2.0092$. 


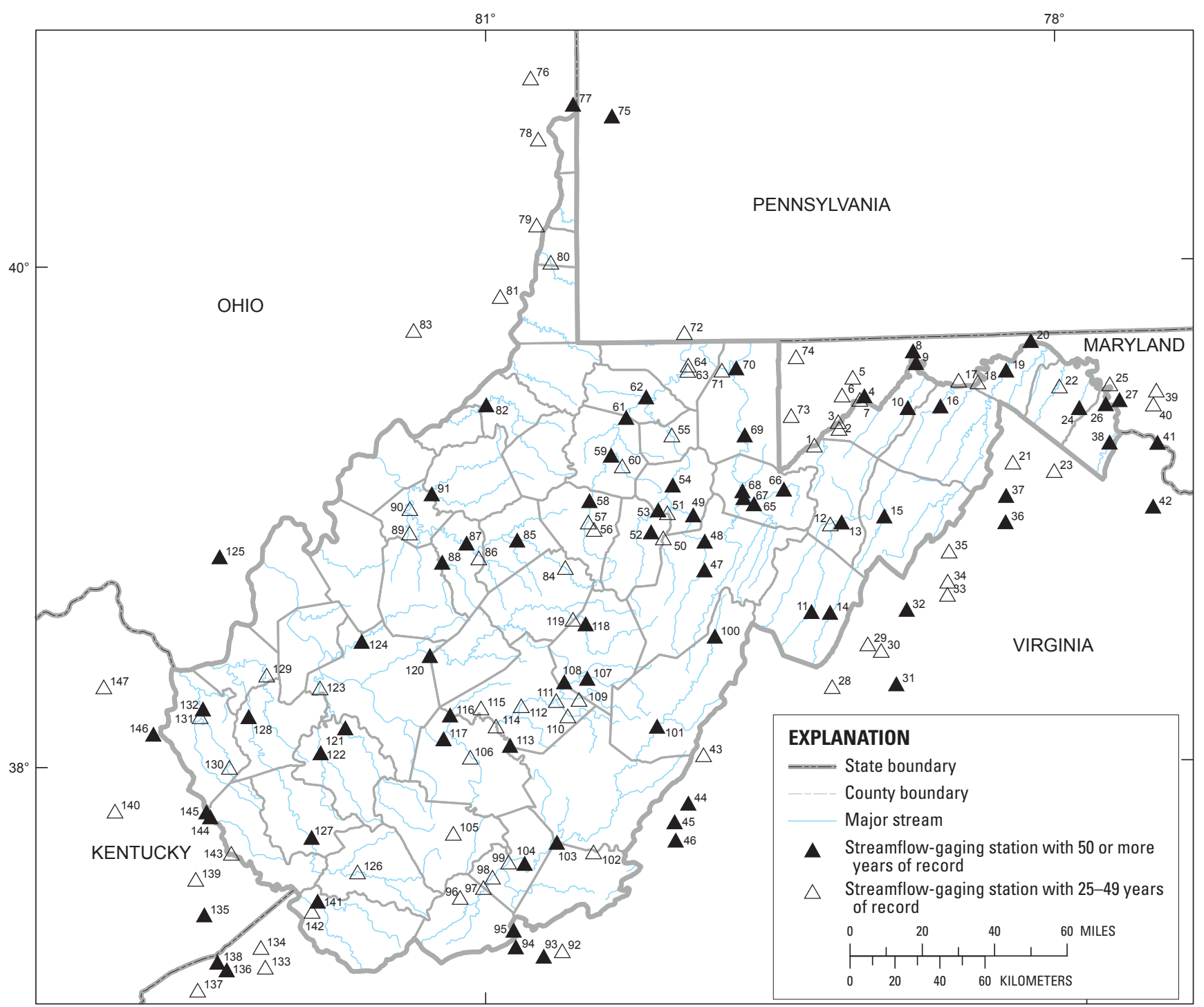

Base from U.S. Geological Survey 1:2,000,000-scale state and county boundaries of the conterminous United States, 1994;

1:2,000,000 coverage of streams for the conterminous United States, 1991. Universal Transverse Mercator projection, zone 17, NAD 83.

Figure 1. Location of U.S. Geological Survey streamflow-gaging stations with 25 or more years of record in or near West Virginia. (Identification numbers refer to stations listed in table 1.) 
maps_diagrams/regional_skewness_map.gif, has been used since its development as the means of selecting skew coefficients for use in predicting peak flows in West Virginia (U.S. Interagency Advisory Committee on Water Data, 1982). The nationwide map was developed by contouring station skew values plotted at the latitude and longitude of the streamflowgaging station. Basin-centroid locations were not available for use in developing the Bulletin 17B skew map. The West Virginia generalized skew map, presented in this report, was developed using basin centroids rather than station locations.

In general, bias is removed from MSE by subtracting the degrees of freedom (DF) from $\mathrm{N}$ (in other words, the variance is typically used instead of MSE). However, this is not a factor in the Bulletin 17B methodology, thus allowing comparison of MSEs for contouring, averages, and regression analysis (whether or not the DF is known). For instance, in the case of the West Virginia generalized skew map, the DF is unknown because if smoothing were eliminated, the grid would touch each of the 147 data points, yielding zero total error-an absurd result. In lieu of applying a rule, a cautious minimum smoothing limit was used.

\section{Contouring Station Skew Coefficients}

Using the irregular grid of the 75 station skews as Z-values and basin centroids as $\mathrm{X}$ - and Y-points, a generalized regular grid was calculated; it consisted of 11,011 points, made up of 91 latitudes ( 36.5 to 41 degrees, by 0.05 -degree increments) by 121 longitudes (-83.5 to -77.5 degrees, by 0.05 -degree increments). The grid extent was chosen to be large enough so that the centroid of all basins for streamflow-gaging stations in or near West Virginia that are potentially representative of peak streamflows applicable to the State would fall within the grid. The SAS procedure G3GRID was used with the SPLINE option and with the parameter SMOOTH=0.1 (SAS Institute Inc., 1999). G3GRID creates a data set whose horizontal (X and $\mathrm{Y}$ ) variable values form a complete and regular grid, and interpolates and smooths the values of the vertical $(Z)$ variable for each point specified in the X-Y plane. SAS Institute Inc. (1999) includes the major equations of the G3GRID algorithms and explains: "The surface that is generated can be thought of as one that would be formed if a stiff, thin metal plate were forced through or near the given data points***. A smoothed spline trades closeness to the original data points for smoothness. To find a value that produces the best balance between smoothness and fit to the original data, you can try several values for the SMOOTH= option."

The 11,011 X-Y points were then projected to UTM (Universal Transverse Mercator, zone 17, NAD 27) and, along with Z-values, were used to construct a Triangulated Irregular Network (TIN) using the following software: ArcInfo 9.2 and ArcMap 9.2, and ArcView 3.3 (Environmental Systems Research Institute, Inc., 2006 and 2002, respectively). Digitally and conceptually, the skew map exists as a TIN (with interpolation provided to get a Z-value for any X-and Y-value). A shapefile of contour lines was generated using the TIN Contour Tool of the 3D Analyst from the ArcMap software then re-projected to Universal Transverse Mercator, zone 17, NAD 83. The contour lines can be made at any selected contour interval from this conceptual skew map; a contour interval of 0.1 is displayed in figure 2. The MSE of the West Virginia generalized skew map, developed from the 147 streamflow-gaging-station skews, is 0.2174 (fig. 2).

\section{Regional Averages}

Average station skews for test regions and the resulting regional MSE for the full 147-station data set are presented in table 2. Data in the column labeled "average of systematic skews" are optimum means for the full 147-station data set, meaning that these optimums cannot be excelled by the means of any subset. The best result (lowest MSE) was obtained using the means of four regions (described in the first row of table 2), yielding a MSE of 0.2546 .

\section{Regional Regression Analysis}

A screening was done to evaluate the relations of 38 basin variables (Paybins, 2008) to the station skew. Results are presented in table 3 .

\section{One-Variable Results}

The single best descriptive variable is annual snowfall (SNOW), with a coefficient of determination, $\mathrm{r}^{2}$, of 0.303 (0.294 adjusted for degrees of freedom). However, this may not mean that snow itself is the best describer of skew. Mean elevation (E) is the next best descriptive variable, with $\mathrm{r}^{2}$ of 0.168 ( 0.156 adjusted for degrees of freedom). SNOW may be proportional to an important effect of the topography that E fails to describe. The 147-site MSE for the SNOW model is 0.2334 for the equation:

$$
\text { SKEW }=-0.4730142+0.0164339 \text { SNOW, }
$$

where

SKEW is the station skew (unitless), and

SNOW is the annual average snowfall in inches.

SNOW is an annual average of map-grid snow values that fall within each drainage basin. The reference used by Paybins (2008) is no longer available. A readily accessible mean total snowfall for the "Lower 48 States" (SNOW14) was used. SNOW14 signifies the specific methodology and the specific data set explained in U.S. Department of Commerce (1999). A national map of SNOW14 is available at URL http://gis.ncdc. noaa.gov/website/ims-climatls/ussnow14/viewer.htm (U.S. Department of Commerce, 2008). This method differs from that used in previous flood studies in West Virginia, including Wiley and others (2000), in which SNOW was determined by 


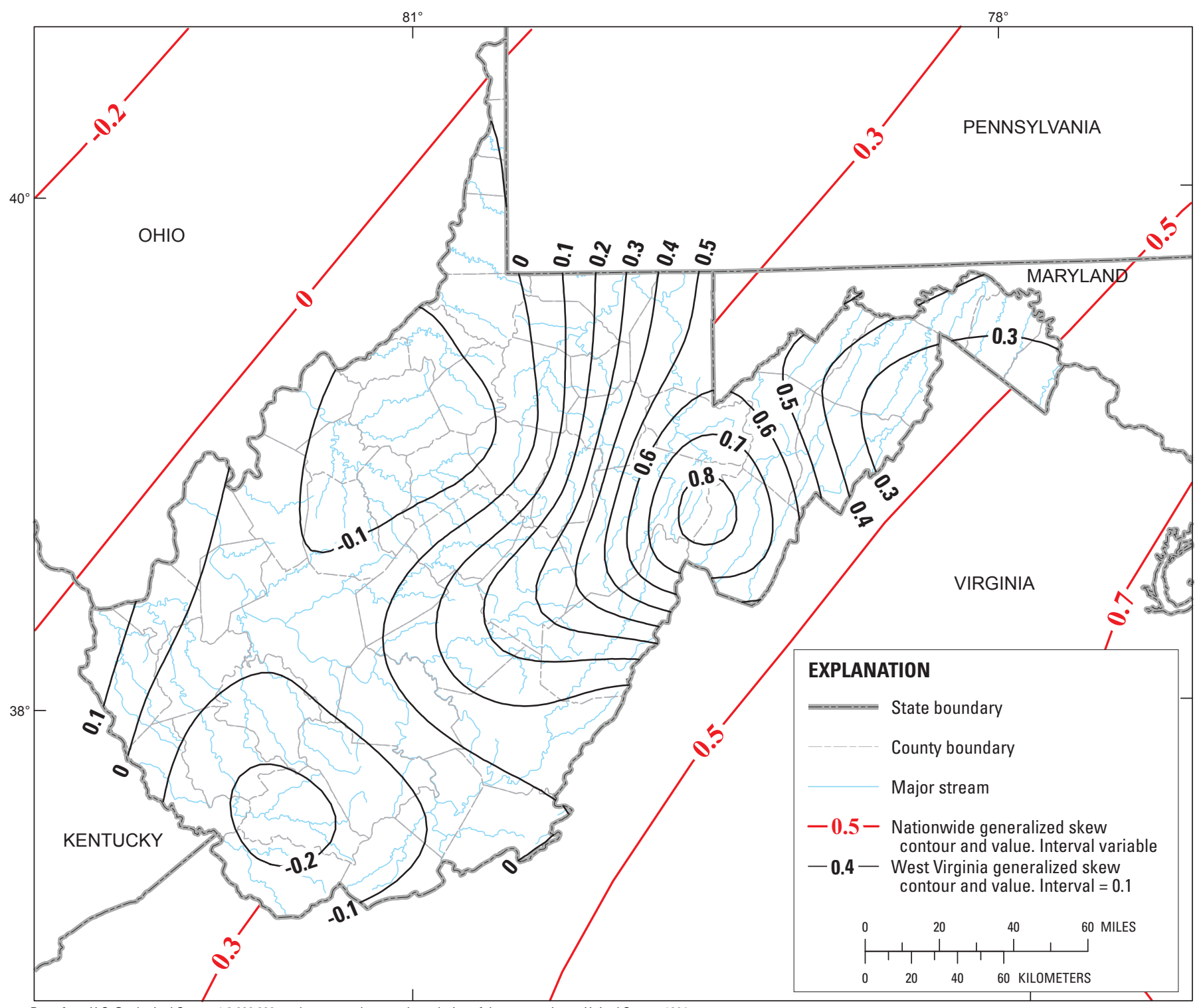

Base from U.S. Geological Survey 1:2,000,000-scale state and county boundaries of the conterminous United States, 1994; 1:2,000,000 coverage of streams for the conterminous United States, 1991. Universal Transverse Mercator projection, zone 17, NAD 83.

Figure 2. Generalized skew map of West Virginia. (Nationwide generalized skew refers to Generalized Skew Coefficients of Logarithms of Annual Streamflow, Bulletin 17B of the Hydrology Subcommittee, Interagency Advisory Committee on Water Data, 1982.) 
Table 2. Average station skews and mean square errors for test regions using the 147 streamflow-gaging stations in or near West Virginia.

$\left[\mathrm{mi}^{2}\right.$, square miles]

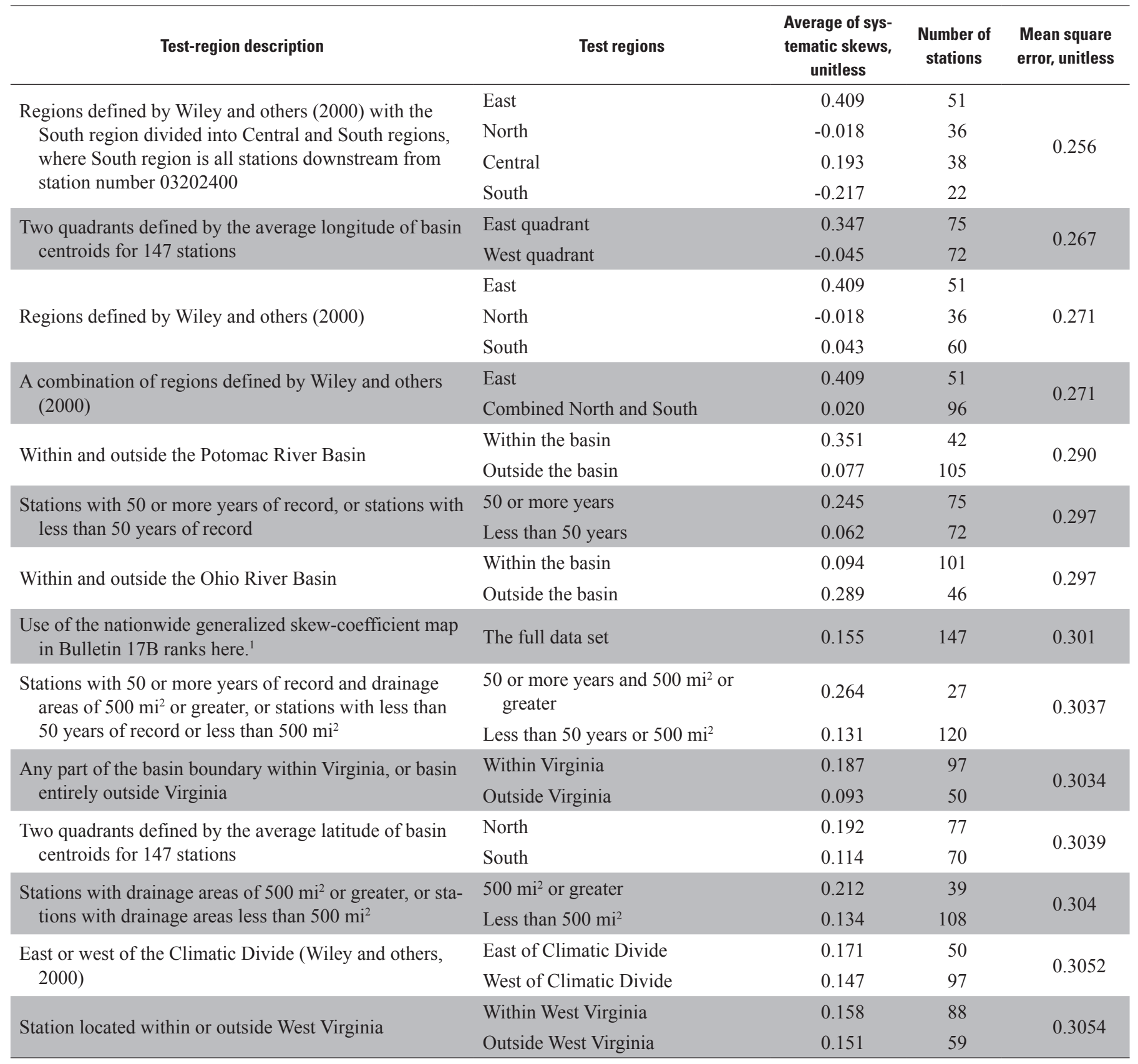

${ }^{1}$ The pre-existing procedure is to use generalized skew values from the national skew map (Interagency Advisory Committee on Water Data, 1982). Average mean square error for the 147 stations using the national skew map is 0.3014 (the national mean square error is 0.3025 ). 
Table 3. Results of screening relations of 38 basin variables to the generalized skew, determined from the West Virginia generalized skew map, for 147 streamflow-gaging stations using SAS procedure REG (with the "by r" option).

[SAS procedure REG is described in SAS Institute Inc., 1999; $\mathrm{r}^{2}$, correlation coefficient; - -, less than 0.15]

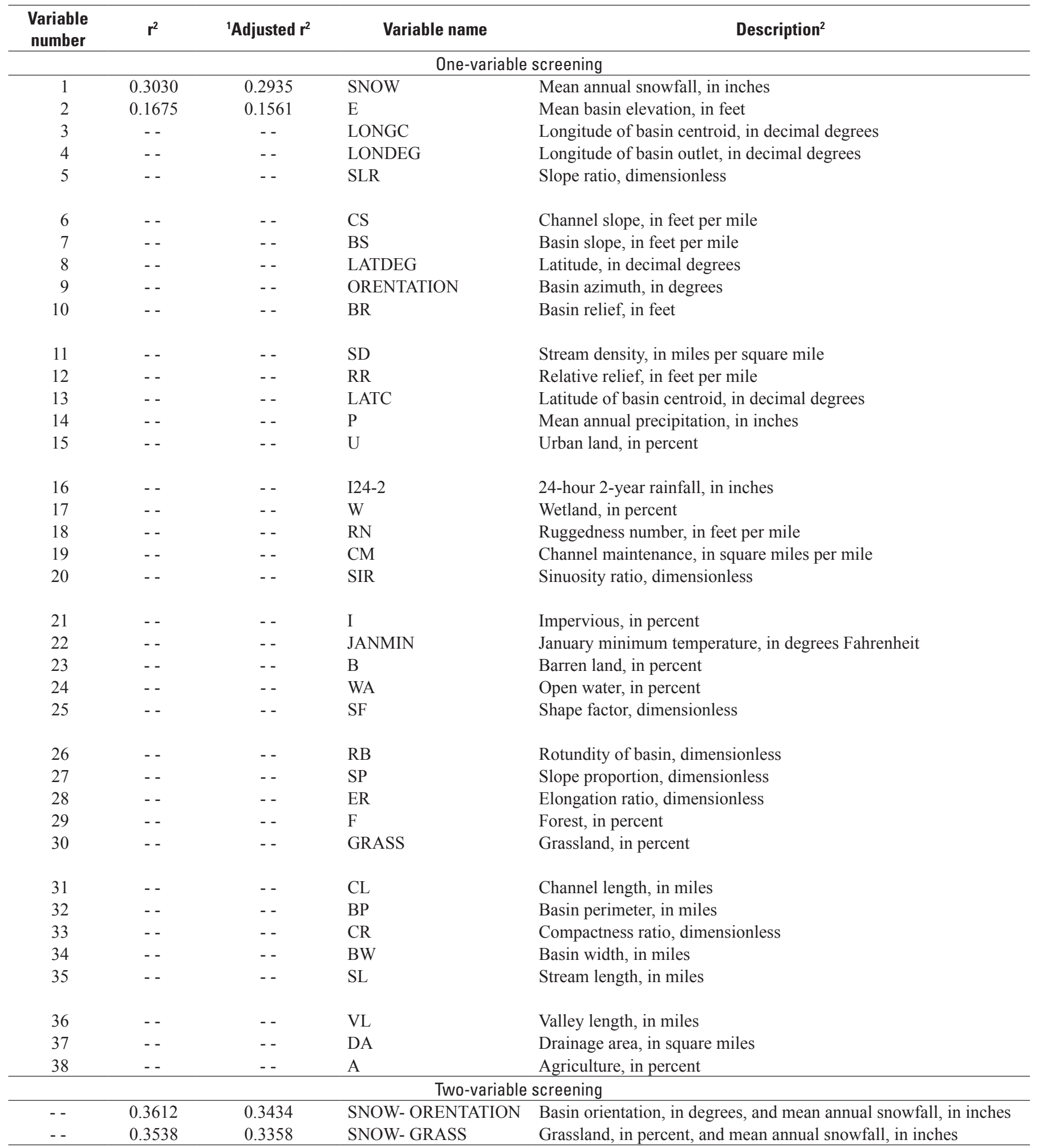

\footnotetext{
${ }^{1}$ Adjusted $\mathrm{r}^{2}$ is an $\mathrm{r}^{2}$ that is adjusted for the number of degrees of freedom in the screening equation and the number of degrees of freedom of the data set.
}

${ }^{2}$ Additional information is available in Paybins (2008). 
visually integrating an isohyetal map published by the U.S. Department of Commerce (1968) within the area of a delineated basin.

\section{Multi-Variable Results}

Only two of the two-variable regression models yielded a 0.05 improvement in $\mathrm{r}^{2}$ beyond the best single-variable model- the SNOW-ORIENTATION and SNOW-GRASS models (table 3). However, no two-variable model gave a 0.05 improvement in adjusted $\mathrm{r}^{2}$. No three-variable model was better than the best two-variable model by more than 0.05 for $\mathrm{r}^{2}$; no four-variable model was better than the best three-variable model by more than 0.05 for $\mathrm{r}^{2}$; and no five-variable model was better than the best four-variable model by more than 0.05 for $\mathrm{r}^{2}$.

The 147-station MSE for the SNOW-ORIENTATION model was 0.2215 , where ORIENTATION (basin average streamflow orientation) was calculated as the orientation of a line averaged from the flow grid, colinear with the direction of flow, as determined by the direction of steepest descent from each cell. ORIENTATION, therefore, is an angle, not an azimuth. ORIENTATION is expressed in degrees, with a possible range of $0^{\circ}$ to $180^{\circ}$, defined as its angle with the $\mathrm{x}$-axis (an east-west or horizontal line). ORIENTATION increases counterclockwise starting at $0^{\circ}$ in the east and going through $90^{\circ}$ when the major axis is vertical, so there is a discontinuity at $0^{\circ}=180^{\circ}$ (at $180^{\circ}$ the function drops suddenly to $0^{\circ}$ ).

The 147-station MSE for the SNOW-GRASS model was 0.2426 where GRASS is a percentage of the area (30-meter grid cells) designated as grassland within a drainage basin. The National Land Cover Database (NLCD 2001) data were used to compute GRASS (Multiple Resolution Land Characteristics Consortium, 2007).

\section{Comparison of Generalization Results}

The 147-station MSE results show (1) the lowest MSE was 0.2174 for the West Virginia generalized contour skew map, (2) the MSE for the SNOW-ORIENTATION model was 0.2215 , (3) the MSE for the SNOW model was 0.2334, (4) the MSE for the SNOW-GRASS model was 0.2426, and (5) merely using regional means for four regions (described in the first row of table 2) yielded a MSE of 0.2546. The MSE of 0.2174 for the West Virginia generalized contour skew map is an improvement over the MSE of 0.3025 for the national map presented in Bulletin 17B. The West Virginia generalized skew map is the best of these estimators and is a reasonable, smoothed contouring of the station skews that meets Bulletin 17B guidelines.

\section{Summary}

In an effort to provide updated information related to flood-frequency calculations, the U.S. Geological Survey (USGS), in cooperation with the West Virginia Department of Transportation, Division of Highways, conducted an investigation to develop generalized skew coefficients for rural, unregulated streams in West Virginia.

A skew coefficient is used to match peak-streamflow data to the log-Pearson Type III statistical distribution. An analysis of records from 147 streamflow-gaging stations in or near West Virginia having a minimum of 25 years of record was used to determine the best method for calculating generalized skew for the State. The procedures for the analysis generally followed guidelines established by the Interagency Advisory Committee on Water Data described in Bulletin 17B.

The difference between the mean skew for a subset of 75 stations having 50 years of record and the mean skew for the 147 stations was statistically significant. Therefore, the analysis of skews was based on the 75 stations rather than the initial 147 stations. The use of stations having 50 or more years of record was a more restrictive exception to the established guidelines.

The generalized-skew analysis included contouring, averaging, and regression of station skews. The best method was determined as the smallest mean square error (MSE), determined by comparing the station value to the regionalized value. The contouring of station skews was the best method for determining generalized skew, with a MSE of 0.2174 . The MSE of 0.2174 is an improvement over the MSE of 0.3025 for the national map presented in Bulletin 17B.

\section{Acknowledgments}

The authors thank colleagues Thomas P. Suro and William H. Asquith for their technical reviews of this report. The authors are also grateful to William P. Bartlett, USGS Surface-Water Specialist for the Northeast and Midwest Areas, for helpful review and comments. The technical assistance of USGS West Virginia Water Science Center colleagues Ronald D. Evaldi and Hugh E. Bevans also is appreciated. 


\section{References Cited}

Environmental Systems Research Institute (ESRI), Inc., 2006, ArcInfo 9.2 and ArcMap 9.2: Redlands, Calif., ESRI, Inc.

Environmental Systems Research Institute (ESRI), Inc., 2002, ArcView 3.3: Redlands, Calif., ESRI, Inc.

Flynn, K.M., Kirby, W.H., and Hummel, P.R., 2006, User's manual for program PeakFQ annual flood-frequency analysis using Bulletin 17B guidelines: U.S. Geological Survey Techniques and Methods, book 4, chap. B4, 42 p., accessed January 17, 2008, at URL http://pubs.usgs.gov/tm/2006/ tm4b4/tm4b4.pdf and http://water.usgs.gov/software/ PeakFQ/

Interagency Advisory Committee on Water Data, 1982, Guidelines for determining flood flow frequency, Bulletin 17B of the Hydrology Subcommittee: Reston, Va., U.S. Geological Survey, Office of Water Data Coordination, 183 p. (available from National Technical Information Service, Springfield, Va., as report no. PB 86157 278, and from Federal Emergency Management Agency at URL http://www.fema. gov/mit/tsd/dl_flow.htm )

Multi-Resolution Land Characteristics Consortium, 2007, National land cover dataset, accessed September 20, 2007, at URL http://www.mrlc.gov/mrlc2k_nlcd.asp

Paybins, K.S., 2008, Basin characteristics for selected streamflow-gaging stations in and near West Virginia: U.S. Geological Survey Open-File Report 2008-1087, 9 p. (available at URL http://pubs.usgs.gov/of/2008/1087/).

SAS Institute Inc., 1990, SAS/STAT user's guide, v. 2, version 6 (4th ed.): Cary, N.C., SAS Institute Inc.
SAS Institute Inc. 1999, SAS/GRAPH® software: Reference, version 8: Cary, N.C., SAS Institute Inc., accessed November 2, 2007, at URL http://www2.stat.unibo.it/ManualiSas/ gref/c30.pdf

Tasker, G.D., and Stedinger, J.R., 1986, Regional skew with weighted LS regression: Journal of Water Resources Planning and Management, v. 112, no. 2, p. 225-237.

U.S. Department of Commerce, 1968, Climatic atlas of the United States: Environmental Data Service, 80 p.

U.S. Department of Commerce, 1999, U.S. snow climatology (Data set documentation TD-9641M): Asheville, N.C., U.S. Department of Commerce, National Oceanic and Atmospheric Administration, National Climatic Data Center, accessed January 30, 2008, at URL http://wwwl ncdc.noaa. gov/pub/data/documentlibrary/tddoc/td9641m.pdf

U.S. Department of Commerce, 2005, Climate maps of the United States: U.S. Department of Commerce, National Oceanic and Atmospheric Association, National Climatic Data Center, accessed January 30, 2008, at URL http://cdo.ncdc.noaa.gov/cgi-bin/climaps/climaps.

pl? directive $=$ order_details $\&$ subrnum $=\&$ region $=$ Lower $\% 20$ 48\%20States \& filename $=$ snow 1413

U.S. Geological Survey, 2008, National Water Information System: Web interface-Peak streamflow for the Nation, accessed January 17, 2008, at URL http://nwis/waterdata. usgs.gov/usa/nwis/peak

Wiley, J.B., Atkins, J.T., Jr., and Tasker, G.D., 2000, Estimating magnitude and frequency of peak discharges for rural, unregulated streams in West Virginia: U.S. Geological Survey Water-Resources Investigations Report 00-4080, 93 p. 
For additional information call or write to:

Director, U.S. Geological Survey

West Virginia Water Science Center

11 Dunbar Street, Charleston, WV 25301

(304) 347-5130

http://wv.usgs.gov 


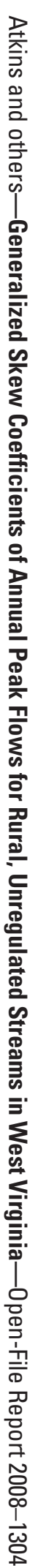

8 Printed on recycled paper 